\title{
Depolarizing differential Mueller matrices
}

\author{
Noé Ortega-Quijano* and José Luis Arce-Diego \\ Applied Optical Techniques Group, Electronics Technology, Systems and Automation Engineering Department, \\ University of Cantabria, Avenida de los Castros S/N, 39005 Santander, Cantabria, Spain \\ *Corresponding author: ortegan@unican.es
}

Received April 18, 2011; revised May 19, 2011; accepted May 23, 2011;

posted May 24, 2011 (Doc. ID 145948); published June 21, 2011

The evolution of a polarized beam can be described by the differential formulation of Mueller calculus. The nondepolarizing differential Mueller matrices are well known. However, they only account for 7 out of the 16 independent parameters that are necessary to model a general anisotropic depolarizing medium. In this work we present the nine differential Mueller matrices for general depolarizing media, highlighting the physical implications of each of them. Group theory is applied to establish the relationship between the differential matrix and the set of transformation generators in the Minkowski space, of which Lorentz generators constitute a particular subgroup. (c) 2011 Optical Society of America

OCIS codes: $260.5430,260.2130,120.5410$.

Among the matricial methods for the study of polarized light interaction with media, Mueller calculus has emerged as a powerful technique due to its ability to deal with partially polarized light and depolarization effects. In addition, the fact that it is based on observables makes it an appropriate approach for experimental applications. It has been used for the study of a wide range of media, namely crystals, atmosphere and oceanic water, optical fibers, and biological tissues [1].

In 1948 Jones proposed the differential formulation of his calculus for the study of the evolution of totally polarized light in anisotropic media [2]. Some years later, an analogous procedure was performed for Mueller calculus, and the differential Mueller-Jones matrices for nondepolarizing anisotropic media were obtained in full parallelism with Jones' approach [3]. The relationship between group theory and Mueller calculus [4] and the use of group theory to derive the differential matrices [5] have been discussed in detail for Jones and MuellerJones matrices, and the feasibility of separating the dichroism and birefringence effects has been demonstrated remarkably [5]. The existence of an infinitesimal generator matrix for a general Mueller matrix has been recently proved for Mueller matrices associated with a definite positive coherency matrix [ 6 ] .

The extension of the differential Mueller calculus to depolarizing media has remained an unresolved issue, and thus the capacity of the differential Mueller calculus to deal with depolarization phenomena is partly underused. In this Letter we present and discuss the differential Mueller matrices for depolarizing media. They complete the set of 16 differential Mueller matrices that fully describe general anisotropic depolarizing media.

First, we consider a fluctuating beam described by the stationary statistical ensemble of its transversal Gaussian-distributed electric plane wave field. We adopt a right-handed Cartesian coordinate system and assume that the beam travels toward the observer with a complex propagation constant $\tilde{\eta}=\eta+i \kappa$. The reference frame is set so that propagation is along the $z$ axis, and thus the field can be resolved into a pair of orthogonal polarization states along $x$ and $y$. Stokes parameters $\left\langle S_{k=0 \ldots 3}\right\rangle$ describe the polarimetric characteristic of the beam in terms of the field observables, which are directly related to the coherency matrix $[\underline{7}, \underline{8}]$. They form the Stokes vector $\overrightarrow{\mathbf{S}}$, which satisfies

$$
d \overrightarrow{\mathbf{S}} / d z=\mathbf{m} \overrightarrow{\mathbf{S}},
$$

where $\mathbf{m}$ is the $4 \times 4$ differential Mueller matrix that describe the polarimetric behavior of an infinitesimal slab of the medium [3]. The differential matrix is related to the macroscopic Mueller matrix by

$$
\mathbf{m}=\lim _{\Delta z \rightarrow 0}\left[\left(\mathbf{M}_{\mathbf{z}, \Delta \mathbf{z}}-\mathbf{I}\right) / \Delta z\right],
$$

where $\mathbf{M}_{\mathbf{z}, \Delta \mathbf{z}}$ is the Mueller matrix that describes the medium from $z$ to $z+\Delta z$, and $\mathbf{I}$ is the $4 \times 4$ identity matrix. Equation (1) establishes a system of four first-order linear differential equations that describe the evolution of the Stokes parameters as a function of $z$. The solution of the system for a linear homogeneous medium between $z_{0}$ and $z$ that verifies $\mathbf{M}_{\mathbf{z}_{0}=\mathbf{0}}=\mathbf{I}$ is

$$
\mathbf{M}=\exp (\mathbf{m} z) .
$$

In general, the behavior of nondepolarizing anisotropic media arises from eight basic properties, which reduce to seven for Mueller calculus as a result of the loss of absolute phase information. Each of them is associated with a basic optical device whose macroscopic Mueller matrix is well defined [1]. Equation (2) enables us to obtain the seven differential Mueller matrices $\mathbf{m}_{\mathbf{n}}$ for each basic type of nondepolarizing optical behavior [3]. Four of them are related to changes in the amplitude of the beam:

$$
\begin{aligned}
& \mathbf{m}_{\mathbf{1}}=\kappa_{i} \mathbf{K}_{\mathbf{i}}=\kappa_{i} \mathbf{I}, \\
& \mathbf{m}_{\mathbf{2}}=\kappa_{q} \mathbf{K}_{\mathbf{q}} \\
& =\kappa_{q}\left[\begin{array}{llllllllllllllll}
0 & 1 & 0 & 0 ; & 1 & 0 & 0 & 0 ; & 0 & 0 & 0 & 0 ; & 0 & 0 & 0 & 0
\end{array}\right] \text {, } \\
& \mathbf{m}_{\mathbf{3}}=\kappa_{u} \mathbf{K}_{\mathbf{u}} \\
& =\kappa_{u}\left[\begin{array}{llllllllllllllll}
0 & 0 & 1 & 0 ; & 0 & 0 & 0 & 0 ; & 1 & 0 & 0 & 0 ; & 0 & 0 & 0 & 0
\end{array}\right] \text {, } \\
& =\kappa_{v}\left[\begin{array}{llllllllllllllll}
0 & 0 & 0 & 1 ; & 0 & 0 & 0 & 0 ; & 0 & 0 & 0 & 0 ; & 1 & 0 & 0 & 0
\end{array}\right] \text {, }
\end{aligned}
$$


which correspond to isotropic absorption (subscript $i$ ), linear dichroism along the $x-y$ axis $(q)$, the bisectors of the $x-y$ axis $(u)$, and circular dichroism $(v)$. The other three are related to phase variations:

$$
\begin{aligned}
& \mathbf{m}_{\mathbf{5}}=\eta_{q} \mathbf{H}_{\mathbf{q}}
\end{aligned}
$$

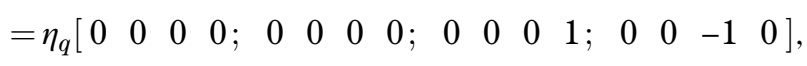

$$
\begin{aligned}
\mathbf{m}_{\mathbf{6}} & =\eta_{u} \mathbf{H}_{\mathbf{u}} \\
& =\eta_{u}\left[\begin{array}{llllllllllllllll}
0 & 0 & 0 & 0 & 0 & 0 & 0 & 1 ; & 0 & 0 & 0 & 0 ; & 0 & -1 & 0 & 0
\end{array}\right],
\end{aligned}
$$

$$
\begin{aligned}
\mathbf{m}_{7} & =\eta_{v} \mathbf{H}_{\mathbf{v}} \\
& =\eta_{v}\left[\begin{array}{llllllllllllllll}
0 & 0 & 0 & 0 & ; & 0 & 0 & 1 & 0 ; & 0 & -1 & 0 & 0 ; & 0 & 0 & 0
\end{array}\right],
\end{aligned}
$$

which account for linear $x-y$, linear $\pm 45^{\circ}$, and circular birefringence. The differential parameters are directly related to $\tilde{\eta}$ [8]. We have adopted the convention $w_{q, u, v}=$ $\left(w_{x,+45, r c p}-w_{y,-45, l c p}\right) / 2$, where $w$ is either $\eta$ or $\kappa$. The general differential matrix of a nondepolarizing medium is obtained as the sum of these seven matrices:

$$
\mathbf{m}^{\mathbf{n d}}=\sum_{n=1}^{7} \mathbf{m}_{\mathbf{n}}=\left[\begin{array}{cccc}
\kappa_{i} & \kappa_{q} & \kappa_{u} & \kappa_{v} \\
\kappa_{q} & \kappa_{i} & \eta_{v} & \eta_{u} \\
\kappa_{u} & -\eta_{v} & \kappa_{i} & \eta_{q} \\
\kappa_{v} & -\eta_{u} & -\eta_{q} & \kappa_{i}
\end{array}\right] .
$$

Each parameter weights the contribution of the corresponding effect to the differential matrix of the medium. For nondepolarizing media there is a direct correspondence between the differential Mueller matrix $\mathbf{m}^{\text {nd }}$ and the differential Jones matrix $[\underline{5}, 8]$.

A deeper analysis of differential Mueller matrices can be performed from a distinct point of view. It has been shown that the Stokes vector is a column vector in a four dimensional fictitious Minkowski space [9,10]. Therefore, group theory can be applied to the study of Mueller calculus. In particular, the mathematics of Lorentz transformations is first considered. A $(1 / 2,1 / 2)$ Lorentz transformation is a matrix given by

$$
\Lambda_{(\mathbf{1} / \mathbf{2}, \mathbf{1} / \mathbf{2})}=\exp \left(\sum_{j=1}^{3} \kappa_{j} \mathbf{K}_{\mathbf{j}}+\eta_{j} \mathbf{H}_{\mathbf{j}}\right),
$$

where $\mathbf{K}_{1 \ldots 3}$ and $\mathbf{H}_{1 \ldots 3}$ are the Lorentz generators for boosts and rotations. They form a closed set for Lorentz transformations in the Minkowski space [4]. The remarkable fact is that they are identical to $\mathbf{K}_{\mathbf{q}, \mathbf{u , v}}$ and $\mathbf{H}_{\mathbf{q}, \mathbf{u , v}}$, the basic matrices involved in differential Mueller matrices $\mathbf{m}_{2 \ldots 6}$ defined above (the sign and the presence of the imaginary unit depend on the author). Lorentz transformations operate on Minkowskian vectors by

$$
\overrightarrow{\mathbf{S}}^{\prime}=\Lambda_{(\mathbf{1 / 2 , 1 / 2})} \overrightarrow{\mathbf{S}}
$$

where the four-vector has been denoted as the Stokes vector in order to highlight the parallelism with Mueller matrix transformations. Comparing Eqs. (12) and (13) with Eqs. (3) and (11) enables us to assert that nondepolarizing differential matrices and Lorentz generators are intimately connected. It can be easily demonstrated that the degree of polarization of a light beam remains invariant upon Lorentz transformations [9].

Nondepolarizing Mueller matrices show a one-to-one correspondence with a Jones matrix [11]. However, depolarizing Mueller matrices no longer arise from a single Jones matrix, but from a statistical ensemble of nondepolarizing matrices. In that case, the Mueller matrix can be obtained as a sum of a maximum of four nondepolarizing matrices [11].

A wide number of well-known media produce depolarization by decreasing the degree of polarization of a light beam [8]. This depolarization effect can be modeled by a generic diagonal Mueller matrix

$$
\operatorname{diag}\left[1, \quad 1, \quad 1, \quad \exp \left(-\kappa_{i, v}^{\prime}\right)\right]
$$

which corresponds to the particular case of the modification of the degree of polarization of the light beam [1] by the variation of the last Stokes parameter. The corresponding differential matrices for diagonal depolarization of $\left\langle S_{1}\right\rangle,\left\langle S_{2}\right\rangle$, and $\left\langle S_{3}\right\rangle$ can be obtained from the macroscopic Mueller matrices using Eq. (2), in an analogous way as it was previously performed for nondepolarizing matrices. Therefore, three depolarizing differential matrices are obtained:

$$
\begin{aligned}
& \mathbf{m}_{\mathbf{8}}=\kappa_{i, q}^{\prime} \mathbf{D}_{\mathbf{q}}=\kappa_{i, q}^{\prime} \cdot \operatorname{diag}\left[\begin{array}{llll}
0, & -1, & 0, & 0
\end{array}\right], \\
& \mathbf{m}_{\mathbf{9}}=\kappa_{i, u}^{\prime} \mathbf{D}_{\mathbf{u}}=\kappa_{i, u}^{\prime} \cdot \operatorname{diag}\left[\begin{array}{llll}
0, & 0, & -1, & 0
\end{array}\right], \\
& \mathbf{m}_{\mathbf{1 0}}=\kappa_{i, v}^{\prime} \mathbf{D}_{\mathbf{v}}=\kappa_{i, v}^{\prime} \cdot \operatorname{diag}\left[\begin{array}{llll}
0, & 0, & 0, & -1
\end{array}\right] .
\end{aligned}
$$

These matrices are the differential Mueller matrices for the three types of diagonal depolarization. The generic differential parameters $\kappa_{i, q}^{\prime}, \kappa_{i, u}^{\prime}$, and $\kappa_{i, v}^{\prime}$ thus model anomalous isotropic absorption of Stokes parameters $\left\langle S_{1}\right\rangle,\left\langle S_{2}\right\rangle$, and $\left\langle S_{3}\right\rangle$, respectively.

Regarding group theory, the basic $\mathbf{D}_{\mathbf{q}, \mathbf{u}, \mathbf{v}}$ matrices associated with $\mathbf{m}_{\mathbf{8}, 9,10}$ differential matrices are the generators for diagonal depolarization. The commutation of $\mathbf{D}_{\mathbf{q}, \mathbf{u}, \mathbf{v}}$ with the Lorentz generators and the isotropic absorption generator $\mathbf{K}_{\mathbf{i}}$ enables us to obtain the six additional generators:

$$
\mathbf{K}_{\mathbf{q}}^{\prime}=\left[\begin{array}{llllllllllllllll}
0 & 1 & 0 & 0 ; & -1 & 0 & 0 & 0 ; & 0 & 0 & 0 & 0 ; & 0 & 0 & 0 & 0
\end{array}\right],
$$

$\mathbf{K}_{\mathbf{u}}^{\prime}=\left[\begin{array}{llllllllllllllll}0 & 0 & 1 & 0 ; & 0 & 0 & 0 & 0 ; & -1 & 0 & 0 & 0 ; & 0 & 0 & 0 & 0\end{array}\right]$,

$$
\mathbf{K}_{\mathbf{v}}^{\prime}=\left[\begin{array}{llllllllllllllll}
0 & 0 & 0 & 1 ; & 0 & 0 & 0 & 0 ; & 0 & 0 & 0 & 0 ; & -1 & 0 & 0 & 0
\end{array}\right],
$$

$\mathbf{H}_{\mathbf{q}}^{\prime}=\left[\begin{array}{llllllllllllllll}0 & 0 & 0 & 0 ; & 0 & 0 & 0 & 0 ; & 0 & 0 & 0 & 1 ; & 0 & 0 & 1 & 0\end{array}\right]$, 


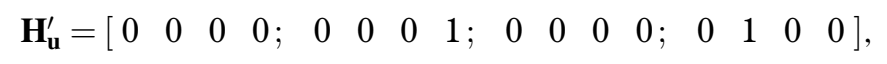

$\mathbf{H}_{\mathbf{v}}^{\prime}=\left[\begin{array}{llllllllllllllll}0 & 0 & 0 & 0 ; & 0 & 0 & 1 & 0 ; & 0 & 1 & 0 & 0 ; & 0 & 0 & 0 & 0\end{array}\right]$.

The whole group of $\mathbf{K}_{\mathbf{i}}, \mathbf{K}_{\mathbf{q}, \mathbf{u}, \mathbf{v}}, \mathbf{H}_{\mathbf{q , u , v}, \mathbf{v}}, \mathbf{D}_{\mathbf{q , u , v}, \mathbf{v}}, \mathbf{K}_{\mathbf{q}, \mathbf{u}, \mathbf{v}}^{\prime}$, and $\mathbf{H}_{\mathbf{q}, \mathbf{u}, \mathbf{v}}^{\prime}$ forms a closed set of 16 generators. However, we have not discussed the physical significance of the last six depolarization generators yet.

In order to gain an insight into the physical implications of these matrices, we consider a nondeterministic medium that presents stochastic fluctuations in its anisotropic properties. This type of medium has been previously studied [4]. Specifically, a birefringent medium with spatial fluctuations in the birefringence magnitude is analyzed. In this particular example it is assumed that the birefringence directors coincide with the Cartesian reference frame and do not vary. If the fluctuation is a purely random Gaussian process, it can be shown that the Mueller matrix that contains the information about fluctuations is given by the following expression:

$$
\mathbf{G}=\left[\begin{array}{cccc}
1 & 0 & 0 & 0 \\
0 & A+a_{q}^{2}(1-A) & a_{q} a_{u}(1-A) & a_{q} a_{v}(1-A) \\
0 & a_{q} a_{u}(1-A) & A+a_{u}^{2}(1-A) & a_{u} a_{v}(1-A) \\
0 & a_{q} a_{v}(1-A) & a_{u} a_{v}(1-A) & A+a_{v}^{2}(1-A)
\end{array}\right],
$$

where $A$ is a constant that depends both on the medium thickness and on the statistical parameters of birefringence variations, while $a_{q}, a_{u}$, and $a_{v}$ are the components of the birefringence constant unit vector [4]. If we obtain the corresponding differential Mueller matrix $\mathbf{g}$, it is observed that it shows six differential parameters in the positions indicated by $\mathbf{D}_{\mathbf{q}, \mathbf{u}, \mathbf{v}}$ and $\mathbf{H}_{\mathbf{q}, \mathbf{u}, \mathbf{v}}^{\prime}$. A similar procedure can be developed for dichroism, which gives rise to the presence of additional differential parameters in the positions indicated by $\mathbf{D}_{\mathbf{q}, \mathbf{u}, \mathbf{v}}$ and $\mathbf{K}_{\mathbf{q}, \mathbf{u}, \mathbf{v}}^{\prime}$. These considerations demonstrate that spatial fluctuations in the medium result in the presence of depolarizing components in the differential Mueller matrix. The presence of temporal randomness produces an analogous effect. A particular example of such a situation is given in [12] for temporal perturbations in the molecular ordering of the inner structure of a cholesteric liquid crystal. In addition, the contribution of the depolarizing matrices proposed in this work can also be observed in other types of media, such as biological Bouligand structures and cholesteric liquid crystal blue phases [13].

Subsequently, $\mathbf{H}_{\mathbf{q}, \mathbf{u}, \mathbf{v}}^{\prime}$ and $\overline{\mathbf{K}}_{\mathbf{q}, \mathbf{u}, \mathbf{v}}^{\prime}$ constitute valid generators for depolarization effects that correspond to specific physical phenomena, which can be described as anomalous birefringence and anomalous dichroism. A simple inspection reveals their close similarity to $\mathbf{H}_{\mathbf{q}, \mathbf{u}, \mathbf{v}}$ and $\mathbf{K}_{\mathbf{q}, \mathbf{u}, \mathbf{v}}$, but with inverted symmetries (symmetric components become skew-symmetric, and vice versa). This aspect was postulated by Azzam to be the main characteristic that should be shown by depolarizing differential Mueller matrices [3] .

The previous considerations result in the following differential Mueller matrices for anomalous birefringence and dichroism:

$$
\begin{aligned}
& \mathbf{m}_{11}=\kappa_{q}^{\prime} \mathbf{K}_{\mathbf{q}}^{\prime}, \\
& \mathbf{m}_{12}=\kappa_{u}^{\prime} \mathbf{K}_{\mathbf{u}}^{\prime}, \\
& \mathbf{m}_{13}=\kappa_{v}^{\prime} \mathbf{K}_{\mathbf{v}}^{\prime}, \\
& \mathbf{m}_{14}=\eta_{q}^{\prime} \mathbf{H}_{\mathbf{q}}^{\prime}, \\
& \mathbf{m}_{15}=\eta_{u}^{\prime} \mathbf{H}_{\mathbf{u}}^{\prime}, \\
& \mathbf{m}_{16}=\eta_{v}^{\prime} \mathbf{H}_{\mathbf{v}}^{\prime},
\end{aligned}
$$

where the differential parameters $\kappa_{q, u, v}^{\prime}$ and $\eta_{q, u, v}^{\prime}$ are given in a generic form. Therefore, the complete set of 16 basic differential Mueller matrices has been obtained. The nondepolarizing effects are contained in seven of them, while the nine differential matrices proposed in this work correspond to depolarizing phenomena. Consequently, the most general differential Mueller matrix for depolarizing anisotropic media is

$$
\mathbf{m}=\sum_{n=1}^{16} \mathbf{m}_{\mathbf{n}}=\left[\begin{array}{cccc}
\kappa_{i} & \kappa_{q}+\kappa_{q}^{\prime} & \kappa_{u}+\kappa_{u}^{\prime} & \kappa_{v}+\kappa_{v}^{\prime} \\
\kappa_{q}-\kappa_{q}^{\prime} & \kappa_{i}-\kappa_{i, q}^{\prime} & \eta_{v}+\eta_{v}^{\prime} & \eta_{u}+\eta_{u}^{\prime} \\
\kappa_{u}-\kappa_{u}^{\prime} & -\eta_{v}+\eta_{v}^{\prime} & \kappa_{i}-\kappa_{i, u}^{\prime} & \eta_{q}+\eta_{q}^{\prime} \\
\kappa_{v}-\kappa_{v}^{\prime} & -\eta_{u}+\eta_{u}^{\prime} & -\eta_{q}+\eta_{q}^{\prime} & \kappa_{i}-\kappa_{i, v}^{\prime}
\end{array}\right] .
$$

The results of this work are of particular interest for the comprehensive study of depolarizing effects in light propagation through samples such as turbid media, liquid crystals, optical fibers, Bragg gratings, and biological tissues $[14,15]$. It is worth noting that the complete set of matrices proposed in this work span all the $4 \times 4$ real matrices, from which physically realizable Mueller matrices are a particular subset [10].

The depolarizing differential Mueller matrices proposed in this Letter enable us to establish a theoretical basis for the application of the general differential Mueller calculus to a vast range of theoretical and experimental applications in many fields of interest in optics.

\section{References}

1. D. Goldstein, Polarized Light (Marcel Dekker, 2003).

2. C. R. Jones, J. Opt. Soc. Am. 38, 671 (1948).

3. R. M. A. Azzam, J. Opt. Soc. Am. 68, 1756 (1978).

4. F. Muhammad and C. S. Brown, Proc. SPIE 2265, 337 (1994).

5. R. Barakat, J. Opt. Soc. Am. A 13, 158 (1996).

6. V. Devlaminck, J. Opt. Soc. Am. A 27, 1529 (2010).

7. L. Mandel and E. Wolf, Optical Coherence and Quantum Optics (Cambridge University, 1995).

8. C. Brosseau, Polarized Light (Wiley, 1998).

9. D. Han, Y. S. Kim, and M. E. Noz, Phys. Rev. E 56 , 6065 (1997).

10. R. Sridhar and R. Simon, J. Mod. Opt. 41, 1903 (1994).

11. S. R. Cloude, Optik 75, 26 (1986).

12. C. Brosseau, Opt. Lett. 20, 1221 (1995).

13. R. Ossikovski, M. Foldyna, C. Fallet, and A. De Martino, Opt. Lett. 34, 2426 (2009).

14. N. Ortega-Quijano, F. Fanjul-Vélez, I. Salas-García, and J. L. Arce-Diego, Proc. SPIE 7906, 790612 (2011).

15. N. Ortega-Quijano and J. L. Arce-Diego, Opt. Lett. 36, 1942 (2011). 